\title{
Management of deep caries lesions with or without pulp involvement in primary teeth: a systematic review and network meta-analysis
}

Tamara Kerber TEDESCO(a)

Thais Marchezini REIS(b)

Anna Carolina Volpi MELLO-MOURA ${ }^{(c)}$

Gabriela Seabra DA SILVA(b)

Samanta SCARPINI(a)

Isabela FLORIANO(d) (iD

Thais GIMENEZ(a)

Fausto Medeiros MENDES(b)

Daniela Prócida RAGGIO(b)

(a) Universidade Ibirapuera - UNIB, Graduate Program in Dentistry, São Paulo, SP, Brazil.

(b) Universidade de São Paulo - USP, School of Dentistry, Department of Orthodontics and Pediatric Dentistry, São Paulo, SP, Brazil.

(c) Universidade Católica Portuguesa, Faculty Dental Medicine, Center for Interdisciplinary Research in Health SalivaTec Lab, Viseu, Portugal.

(d) Centro Universitáciro Uninovafapi, Undergraduate Program in Dentistry, Teresina, PI, Brazil.

Declaration of Interests: The authors certify that they have no commercial or associative interest that represents a conflict of interest in connection with the manuscript.

\section{Corresponding Author:}

Tamara Kerber Tedesco

E-mail: tamara.tedesco@ibirapuera.edu.br

Submitted: Mar 3, 2020

Accepted for publication: July 14, 2020

Last revision: August 4, 2020
Abstract: There is a lack of evidence about the best approach for cavitated caries lesions with the possibility of pulpal involvement in primary teeth. Thus, the present authors aimed to verify the best treatment for deep caries lesions with or without pulp involvement in primary teeth. The search was conducted in MEDLINE/Pubmed and Web of Science databases until May 2020. Studies that compared techniques to manage deep caries lesions with at least 12 months of follow-up were included. The risk of bias was evaluated using the RoB tool. Network meta-analysis and pairwise meta-analyses were conducted considering the treatment clinical success as an outcome, according to the pulp health condition. From 491 potentially eligible studies, 9 were included. For deep caries lesions with pulp vitality, the Hall Technique presented the highest probability of success $(78 \%)$. In the event of accidental pulp exposure, pulpectomy presented a $76 \%$ chance of providing the best clinical results. For pulp necrosis, no difference was observed between a pulpectomy and non-instrumented endodontic treatment ( $R \mathrm{R}=0.69 ; 95 \% \mathrm{CI}$ : 0.21-2.33) Thus, it was concluded that the Hall Technique may be a better option for deep caries lesions with pulp vitality. In cases of accidental pulp exposure of vital teeth during caries removal, a pulpectomy may be considered the best option. However, there are insufficient studies to build up evidence about the best treatment option when irreversible pulpitis or pulp necrosis is present.

Keywords: Dental Caries; Pulpotomy; Pulpectomy; Tooth, Deciduous; Systematic Review.

\section{Introduction}

Despite the implementation of preventive measures for dental caries, data indicate that around $50 \%$ of children still have at least one primary tooth in need of treatment. ${ }^{1}$ The presence of caries lesions, especially the most severe ones, leads to a negative impact on the oral health-related quality of life of patients. ${ }^{2}$ Therefore, their management should be the focus of pediatric dentists.

Some systematic reviews have sought to determine the best treatment for cavitated lesions in primary teeth, concerning both treatment 
success ${ }^{3,4,5}$ and patient-centered outcomes. ${ }^{6}$ However, to date, there is a lack of evidence to establish the best approach for cavitated caries lesions with risk of pulpal involvement in primary teeth.

Most of the available systematic reviews aimed to compare different materials used in the same technique for conservative or non-conservative pulp treatments, $7,8,9$ such as a pulpotomy or pulpectomy. However, the literature indicates more than one treatment option for the same pulpal health diagnosis. ${ }^{9}$ There is no scientifically established approach due to the lack of systematic reviews comparing the numerous treatment options available. A systematic review with network meta-analysis could summarize the results from primary studies. Additionally, it could provide a reliable evidence regarding the effectiveness of treatments to guide dental professionals on the decision-making process when managing cavitated caries lesions with risk of pulpal involvement in primary teeth.

Thus, the objective of the present study was to verify the best treatment for deep caries lesions with or without pulp involvement in primary teeth, considering the pulp health condition, by means of systematic review and network meta-analysis.

\section{Methodology}

\section{Study design}

The present review was performed according to the PRISMA-NMA extension statement, ${ }^{10}$ and was registered in the International Prospective Register of Systematic Reviews (PROSPERO) (protocol \#CRD42016037787; available at http://www.crd.york.ac.uk/PROSPERO/ display_record.php?ID=CRD42016037787). Two reviewers independently performed the entire processes of screening and selecting articles, as well as data collection and risk of bias analyses (TMR and TKT). Certainty of evidence was conducted by two other reviewers independently (TG and TKT). A third researcher, with experience in the treatment of caries lesions in primary teeth (DPR), solved cases of conflict or doubt.

\section{Study sources}

A systematic search was conducted in the MEDLINE / PubMed and Web of Science electronic databases. The grey literature was searched in the OpenGrey database. There was no restriction on the language and the date of publication for study selection. The last search was carried out on May 30, 2020.

\section{Search strategy}

The PICO strategy was used for study selection with the following question: Which treatment shows the highest success rate for deep cavitated caries lesions with or without possible pulpal involvement in primary teeth? (Participants: primary teeth; Intervention: treatments available for deep cavitated caries lesions with or without pulp involvement; Comparator: treatments for deep cavitated caries lesions with or without pulp involvement; Outcome: clinical success of treatment). The search strategy was developed for the MEDLINE / PubMed database and then adapted for the other databases consulted. The results from the different databases were cross-referenced to locate and eliminate duplications. The complete search strategy for MEDLINE / PubMed is shown below:

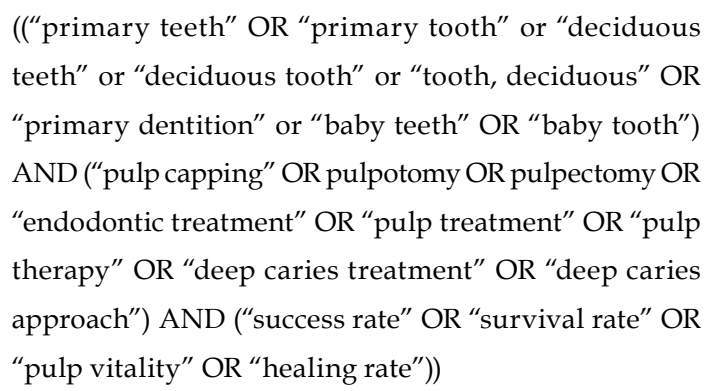

For the Web of Science database, the following strategy was used:

TS=((primary teeth OR primary tooth OR deciduous teeth OR deciduous tooth OR tooth, deciduous OR primary dentition or baby teeth OR baby tooth) AND (pulp capping OR pulpotomy OR pulpectomy OR endodontic treatment OR pulp treatment OR pulp therapy OR deep caries treatment OR deep caries approach) AND (success rate OR survival rate OR pulp vitality OR healing rate))

Finally, the following strategy was used to search in OpenGrey: 
((primary teeth OR primary tooth OR deciduous teeth OR deciduous tooth OR tooth, deciduous OR primary dentition or baby teeth OR baby tooth) AND (pulp capping OR pulpotomy OR pulpectomy OR endodontic treatment OR pulp treatment OR pulp therapy OR deep caries treatment OR deep caries approach) AND (success rate OR survival rate OR pulp vitality OR healing rate))

\section{Eligibility criteria}

Initially, the titles and abstracts of the potentially relevant studies identified in the databases were evaluated independently by two researchers $($ Kappa $=0.8)$. Articles were considered eligible when they met the following inclusion criteria:

a. Evaluated the treatment of deep cavitated caries lesions in primary teeth with or without pulp involvement;

b. Conducted as a prospective study with at least 12 months of follow-up.

After the first evaluation, articles that met the inclusion criteria were reviewed in their entirety, and those which had any of the following exclusion criteria were considered ineligible (Kappa $=0.7$ ):

a. Did not show comparison groups;

b. Evaluated other outcomes not related to the review;

c. Conducted with specific groups of patients (such as patients on medication or with special needs);

d. Did not compare different management techniques.

The reference lists from potentially eligible articles were then screened to check for all relevant articles that may not have been identified during searches in the databases. In cases where more than one article studied the same sample, the one presenting the complete data was considered.

The features of cavitated caries lesionspresence or absence of pulp involvement, reversible or irreversible pulpitis, as well as symptomatic or asymptomatic-were considered according to descriptions of the studies.

\section{Data collection}

The information from eligible studies was collected independently by two reviewers. For each included study, the following data were recorded: initial pulp health diagnosis, publication details (authors and year), sample characteristics (number and age of participants, number of treatments performed per group), study methodology (design, comparative treatments, tooth type, how the pulp diagnosis was performed, clinical characteristics considered for treatment success-outcome), and outcome information (follow-up and clinical success rate of treatment).

\section{Risk of bias assessment}

After the data collection, two researchers independently assessed the possible risk of bias in included studies using the RoB tool ${ }^{11}(\mathrm{Kappa}=0.7)$. The tool considers domains on selection, performance, detection, attrition, and reporting biases. When all domains had a low risk of bias, the study was considered low risk. If at least one domain had an uncertain or high risk of bias, the study received respective ratings.

\section{Evaluation of the certainty of the evidence-GRADE tool}

The GRADE tool was used to evaluate the certainty of evidence of the results from meta-analyses (Kappa $=0.9)$. The certainty of evidence was classified as high, moderate, low, or very low, while the reason for downgrading was based on five domains: study limitations, indirectness, inconsistency, imprecision, and publication bias.

\section{Synthesis of data and statistical methods for network meta-analyses and meta-analyses}

Initially, Cochran $\mathrm{Q}$ and $\mathrm{I}^{2}$ tests were conducted to evaluate the heterogeneity of the data included in the meta-analyses. Meta-analyses were conducted considering clinical success of the treatment as the outcome for cavitated caries lesions with possible pulp involvement according to the initial pulpal health condition. The choice of a fixed or random model was made according to the results of heterogeneity.

Direct evidence was computed through pairwise meta-analyses with random effect when only two treatments were considered (for example, treatment A vs. B). However, when more than 
two treatments were evaluated for the same pulp condition, network meta-analyses were conducted, which synthesize direct and indirect comparisons. For example, when studies compare A vs. B and $B$ vs. $C$, the results from these studies are called direct evidence. Whilst the results from A vs. C, since there are no primary study comparing both treatments, came from indirect evidence from the other studies (A vs. B and B vs. C). The geometry of the networks is composed of nodes, representing the treatment option, which are connected by lines that represent indirect evidence.

To simultaneously consider both direct and indirect evidences, a Bayesian analysis of mixed treatment comparisons (MTC) was carried out. Since all studies were performed with comparable groups of patients, considering similar diagnostic criteria of the initial pulp health condition as well as the protocols of the treatments, this network met the assumption of transitivity. Thus, the MTC analyses were initially conducted using both fixed and random models. The goodness of fit of the models was measured using the residual deviation and the deviation information criterion (DIC). Because the DIC value of the random model was lower, the random-effects model with homogeneous variability between studies was used. A node split analysis for inconsistency was not performed because of insufficient data.

All analyses were performed using R statistical software, version 2.15.3 (R Core Team, 2012, Vienna, Austria). Meta-analyses were conducted using a meta-package, while network meta-analyses were conducted using the GeMTC package and the rJAGS package to estimate the models. The ranking probability of efficacy for all treatments, and the relative risk for pairwise comparisons, odds ratio for mixed comparison, and 95\% confidence intervals were calculated.

\section{Results}

\section{Selection of studies}

The systematic search of the literature identified 489 potentially relevant references, being 173 publications from MEDLINE / PubMed, 315 from the Web of Science, and one from OpenGrey. Of these, 79 were duplicated in more than one database. From the information provided in the title and abstract, 257 articles were considered ineligible. The main reason for non-inclusion was not investigating the treatment of cavitated caries lesions in primary teeth with possible pulp involvement (55.3\%). One hundred and fifty-three remaining papers were analyzed in their entirety for the collection of more detailed information. Thirty-three articles were excluded because they did not present comparison groups (22.6\%), while 109 articles did not compare different techniques (74.7\%). Two publications were identified through manual search. Finally, nine publications met the eligibility criteria and were included in the systematic review. Two studies evaluated similar groups for the treatment of deep caries lesions with pulp vitality, which were not comparable with the other treatments from the other studies. However, one did not present clinical success data and was thus not included in the quantitative analysis. Therefore, seven studies were included in the quantitative analysis. The process of study selection is shown in Figure 1.

\section{Study characteristics}

The main characteristics of the included studies are presented in Table 1.

In general, all studies considered similar characteristics for the initial pulp diagnosis, especially regarding the $\mathrm{X}$-ray images (potential proximity to the pulp, as well as signs of irreversible pulpitis or pulp necrosis). $12,13,14,15,16,17,18,19,20$

Four studies evaluated treatment options for deep caries lesions with pulp vitality in primary molars ${ }^{12,13,14,15}$ and five treatment options were considered: the Hall Technique, selective and nonselective removal of caries lesion, pulpotomy, and pulpectomy. Also, all studies considered clinical signs and symptoms such as spontaneous pain, edema, and fistula, as well as radiographic characteristics as part of the diagnosis of the initial pulp health condition. ${ }^{12,13,14,15}$ From these, three studies considered clinical outcomes for the evaluation of treatment success; ; ${ }^{12,13,15}$ however, one study only reported radiographic success. ${ }^{14}$ The longest follow-up of included studies was 24 months. ${ }^{12,13}$ 


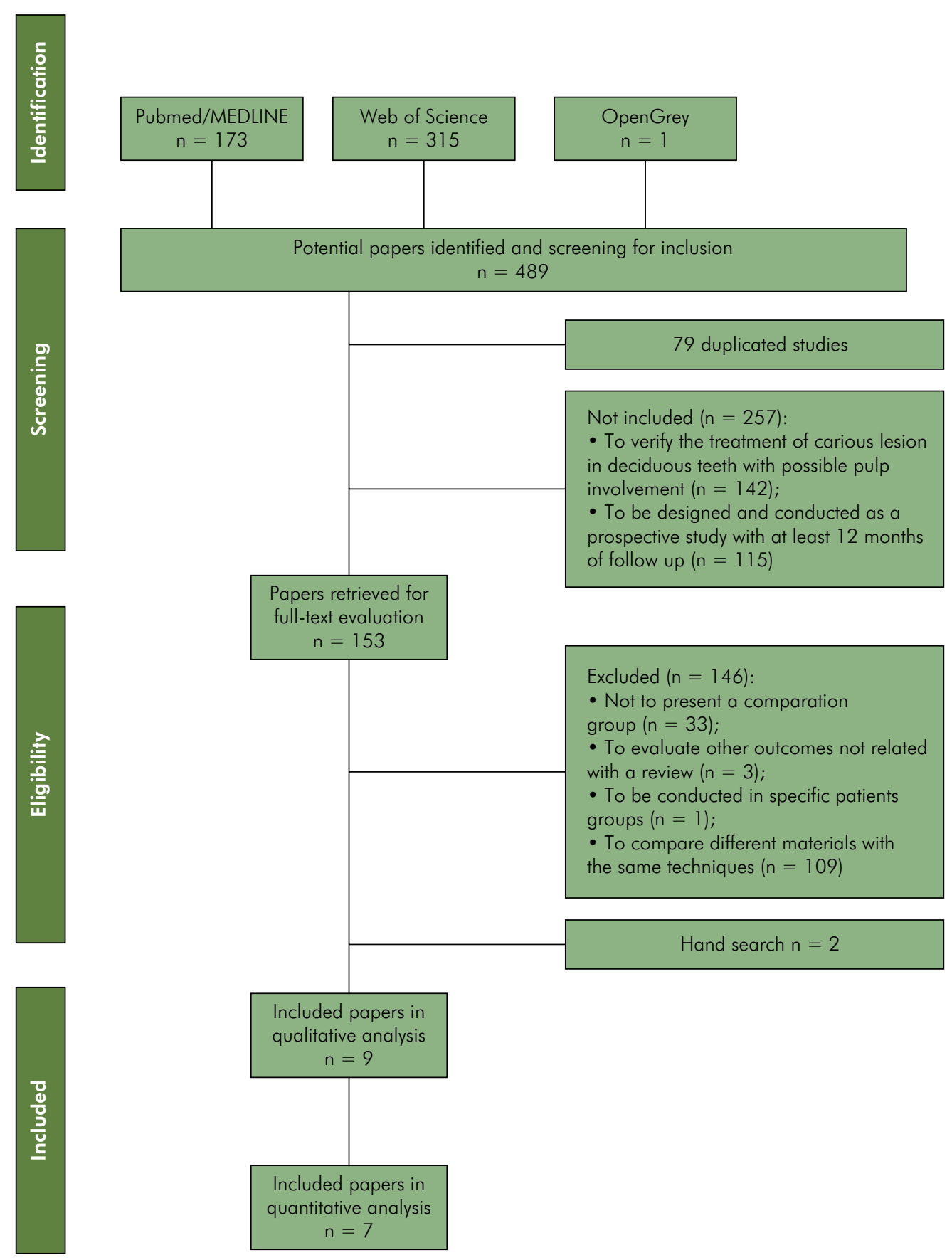

Figure 1. Flowchart of screening, eligibility, and analysis of articles.

Furthermore, three studies considered treatments for primary incisors ${ }^{16,17}$ and molars $^{18}$ with accidental pulp exposure of vital teeth during caries removal. Three treatment options were considered for this pulp condition: direct pulp capping, pulpotomy, and pulpectomy. Only two studies reported clinical and radiographic parameters for the diagnosis of pulp vitality other than lesion depth. ${ }^{16,18}$ Finally, two studies considered clinical outcomes to assess successful treatment in 24 months, ${ }^{16,17}$ and one, in 36 months of follow-up. ${ }^{18}$ 


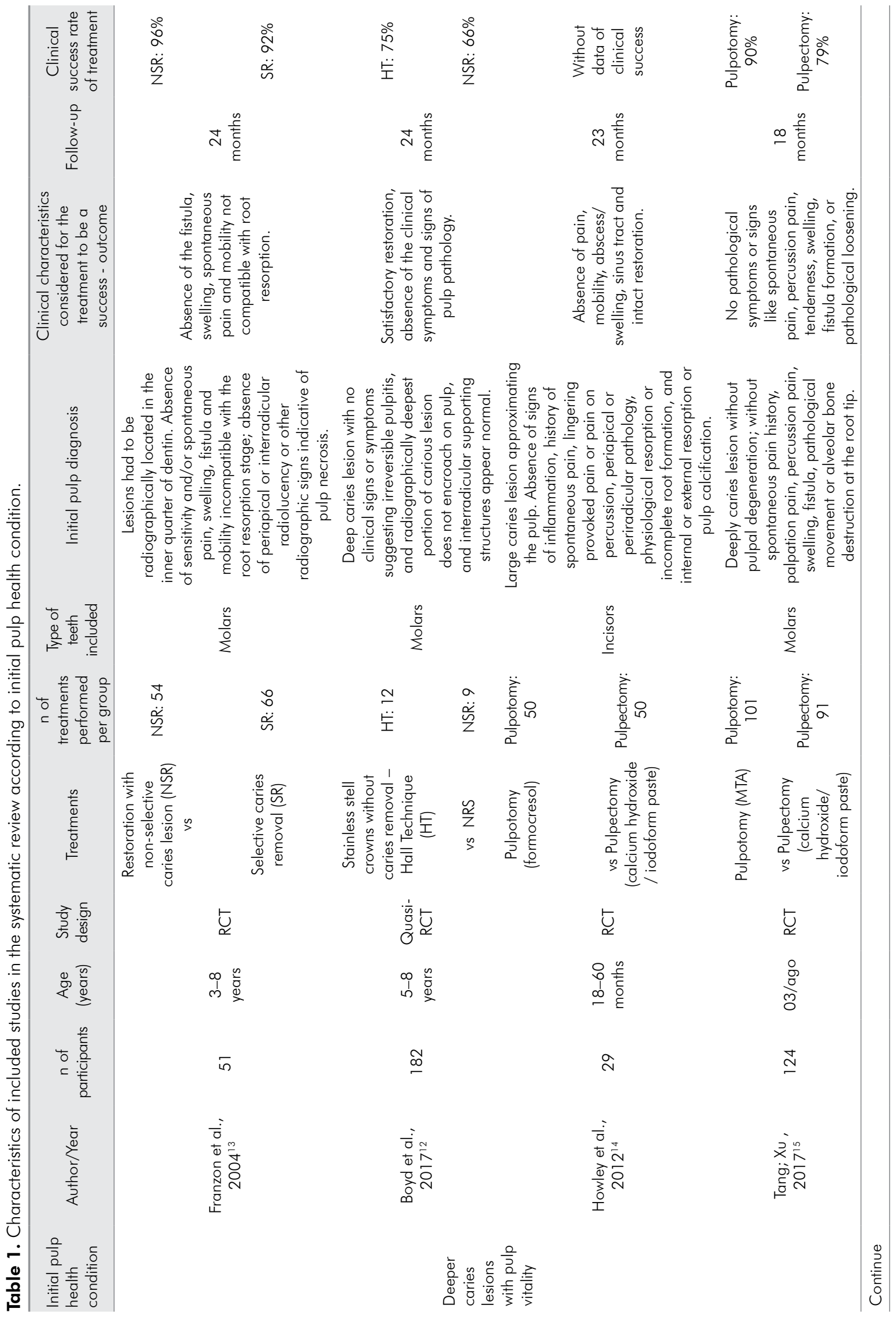




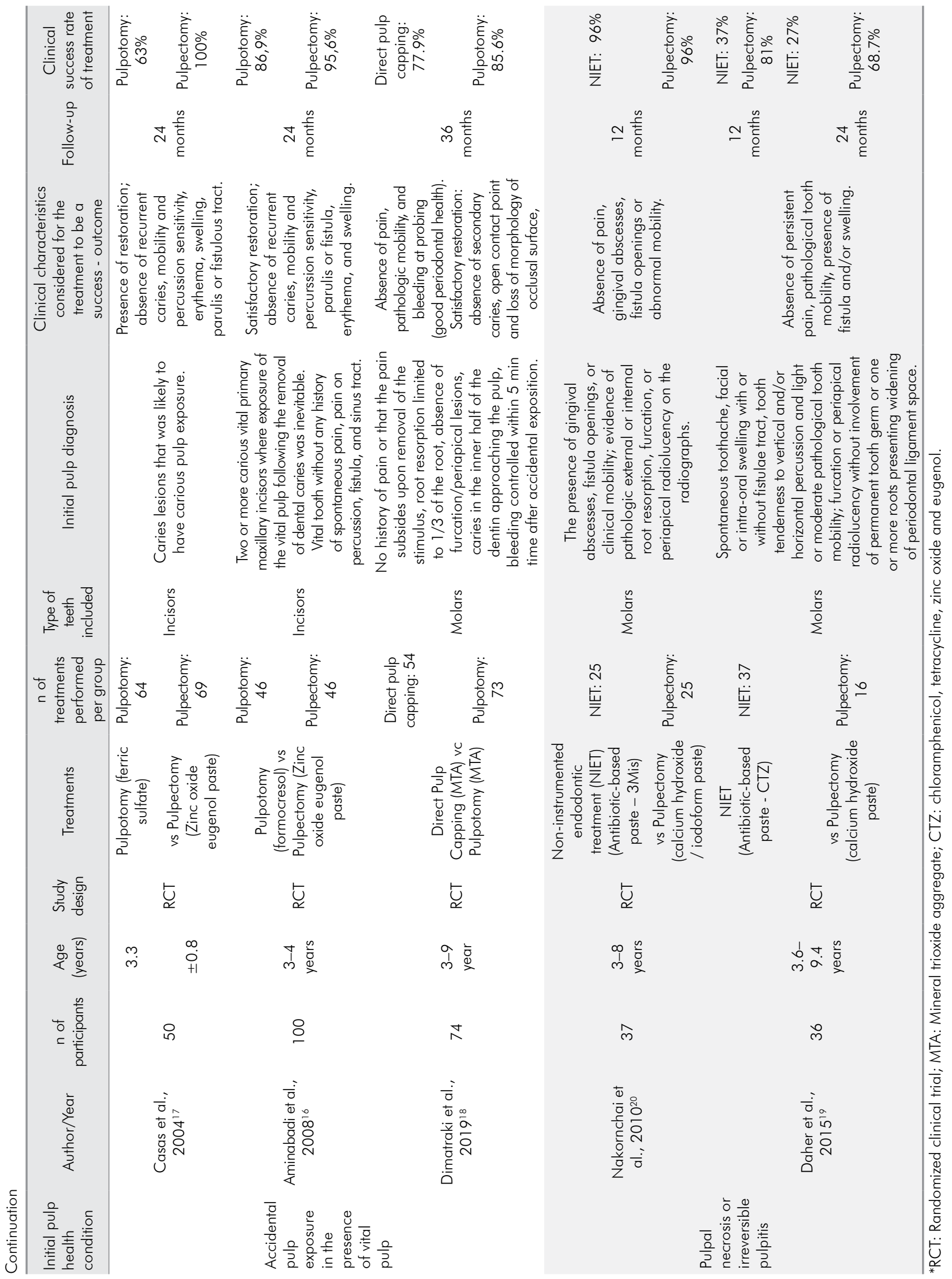


When irreversible pulpitis or pulp necrosis was present, two studies compared two different treatment options for primary molars: pulpectomy (instrumented endodontic treatment) and non-instrumented endodontic treatment (NIET). ${ }^{19,20}$ In general, clinical success was considered as the absence of persistent pain, pathological dental mobility, fistula, or edema. ${ }^{19,20}$ The follow-up time ranged from $12^{20}$ to 24 months. ${ }^{19}$

\section{Risk of bias assessment}

The final analysis of the risk of bias of the included studies is presented in Figure 2. Concerning the selection domain, five articles reported the generation of the randomization sequence ${ }^{13,14,18,19,20}$ and only one used the allocation mechanism. ${ }^{19}$ When performance was considered, two studies reported blinded participants, ${ }^{13,19}$ while for the detection domain, four studies reported the blinding of the evaluators. ${ }^{13,16,17,20}$ However, all the studies presented a low risk of bias for the attrition and reporting domains. No other source of bias was detected. ${ }^{12,13,14,15,16,17,18,19,20}$

In general, all studies had at least one high-risk domain and were considered to have high risk of bias. ${ }^{12,13,14,15,16,17,18,19,20}$

\section{Evaluation of the certainty of evidence}

Table 2 presents the certainty of evidence and the reason for downgrading treatment comparison

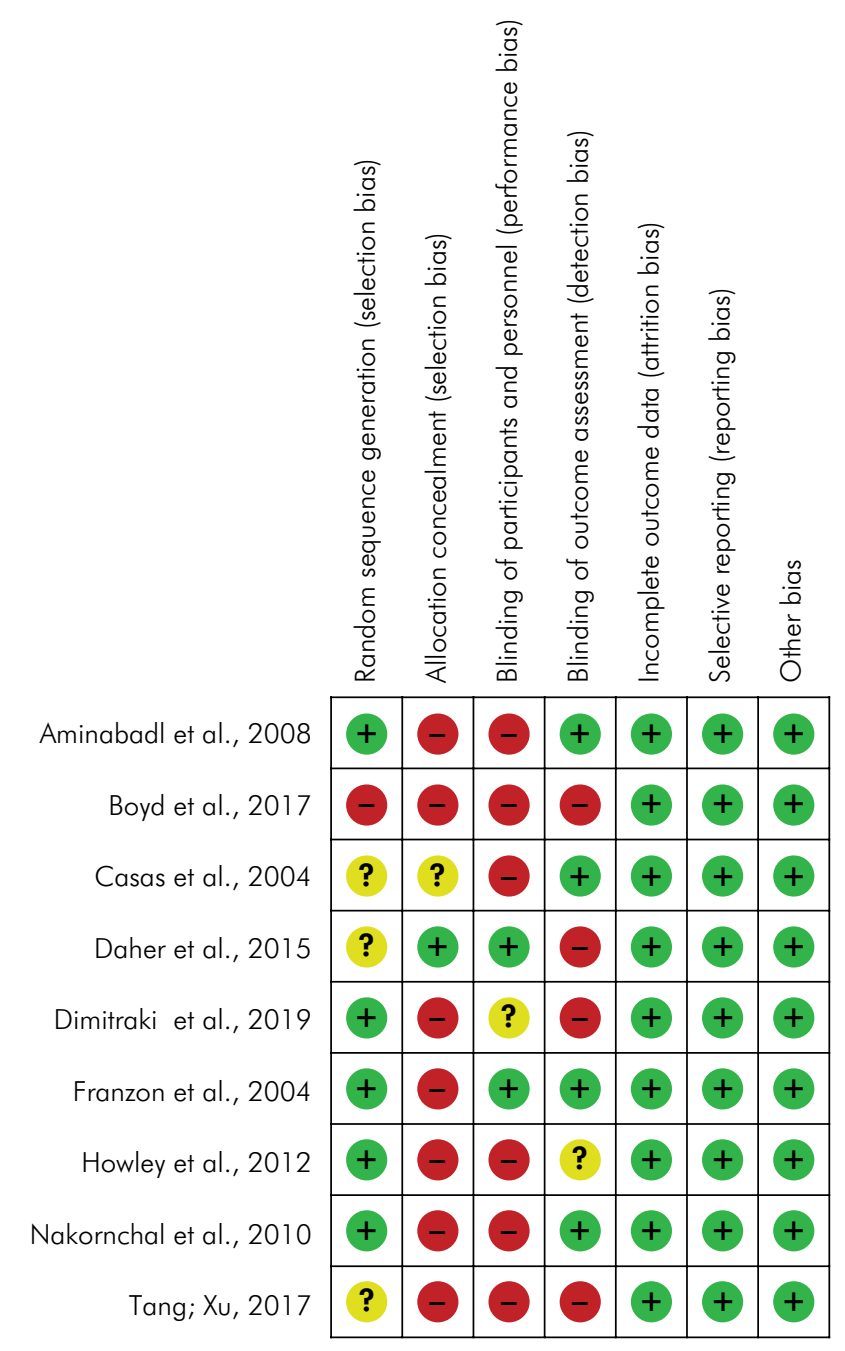

Figure 2. Risk of bias assessment according to the evaluated domain. 
Table 2. Certainty of evidence and reason for downgrading in accordance with the initial pulp health condition.

\begin{tabular}{|c|c|c|}
\hline Comparison & $\begin{array}{l}\text { Certainty of } \\
\text { evidence }\end{array}$ & Reason for downgrading \\
\hline \multicolumn{3}{|l|}{ Deeper caries lesions with pulp vitality } \\
\hline Hall technique vs. Selective caries removal & Very low & Study limitation - High risk of bias; Indirectness; Imprecision. \\
\hline Hall technique vs. Non-selective caries removal & Low & Study limitation - High risk of bias; Imprecision. \\
\hline Selective caries removal vs. Non-selective caries removal & Low & Study limitation - High risk of bias; Imprecision. \\
\hline \multicolumn{3}{|l|}{ Accidental pulp exposure in the presence of vital pulp } \\
\hline Direct pulp capping vs. Pulpotomy & Low & Study limitation - High risk of bias; Imprecision. \\
\hline Direct pulp capping vs. Pulpectomy & Very low & Study limitation - High risk of bias; Indirectness; Imprecision. \\
\hline Pulpotomy vs. Pulpectomy & Moderate & Study limitation - High risk of bias. \\
\hline \multicolumn{3}{|l|}{ Pulpal necrosis or irreversible pulpitis } \\
\hline $\begin{array}{l}\text { Non-instrumented endodontic treatment vs. Instrumented } \\
\text { endodontic treatment }\end{array}$ & Very low & Study limitation - High risk of bias; Inconsistency, Imprecision. \\
\hline
\end{tabular}

Table 3. League table with all mixed* comparisons of treatments for deeper caries lesion with pulp vitality.

\begin{tabular}{ccc}
\hline Hall technique & & \\
$6.2(0.34-140.0)$ & Selective removal of caries lesion & Non-selective removal of caries lesion \\
$2.6(0.31-23.0)$ & $0.43(0.052-2.8)$ & foft
\end{tabular}

in accordance with the initial pulp health condition. In general, the certainty of evidence ranged from very low to moderate. The reasons for downgrading were especially study limitations due to high risk of bias of the primary studies included, indirectness and imprecision.

\section{Data synthesis}

The analyses were conducted in accordance with the pulp health condition. We considered as the outcome the clinical success of the treatments, which was dichotomized as a success or failure according to what was reported in the primary studies.

Thus, two network meta-analyses were performed considering: a) the treatment success rate for deep caries lesions with pulp vitality (Hall Technique, selective caries removal, and non-selective caries removal), and b) the success rate of approaches for vital pulp accidentally exposed during the caries removal (direct pulp capping, pulpotomy, and pulpectomy). Furthermore, one pairwise meta-analysis was conducted considering treatments for irreversible pulpitis or pulp necrosis, comparing instrumented with non-instrumented endodontic treatment.

Data from four studies evaluating deep caries lesions with pulp vitality were initially considered. Two studies evaluated similar groups, not comparable with the others (pulpotomy and pulpectomy). However, one did not present an outcome of clinical success, but only radiographic data. Thus, data from these two studies could not be synthesized. Network meta-analysis was conducted with data from two studies, which evaluated three different treatment options. Low, but not significant, heterogeneity was observed among included studies $\left(\mathrm{p}=1.031 ; \mathrm{I}^{2}\right.$ $=27 \%$ ). The results of the indirect comparisons are presented in Table 3 . The rank probability showed that the best results could be obtained when the Hall Technique was used (78\%) (Figure3), compared to the non-selective and selective removal of caries lesions.

For vital pulp accidentally exposed during the caries removal, three studies were included, and three types of treatment were evaluated and included in the network meta-analysis. Low, but 

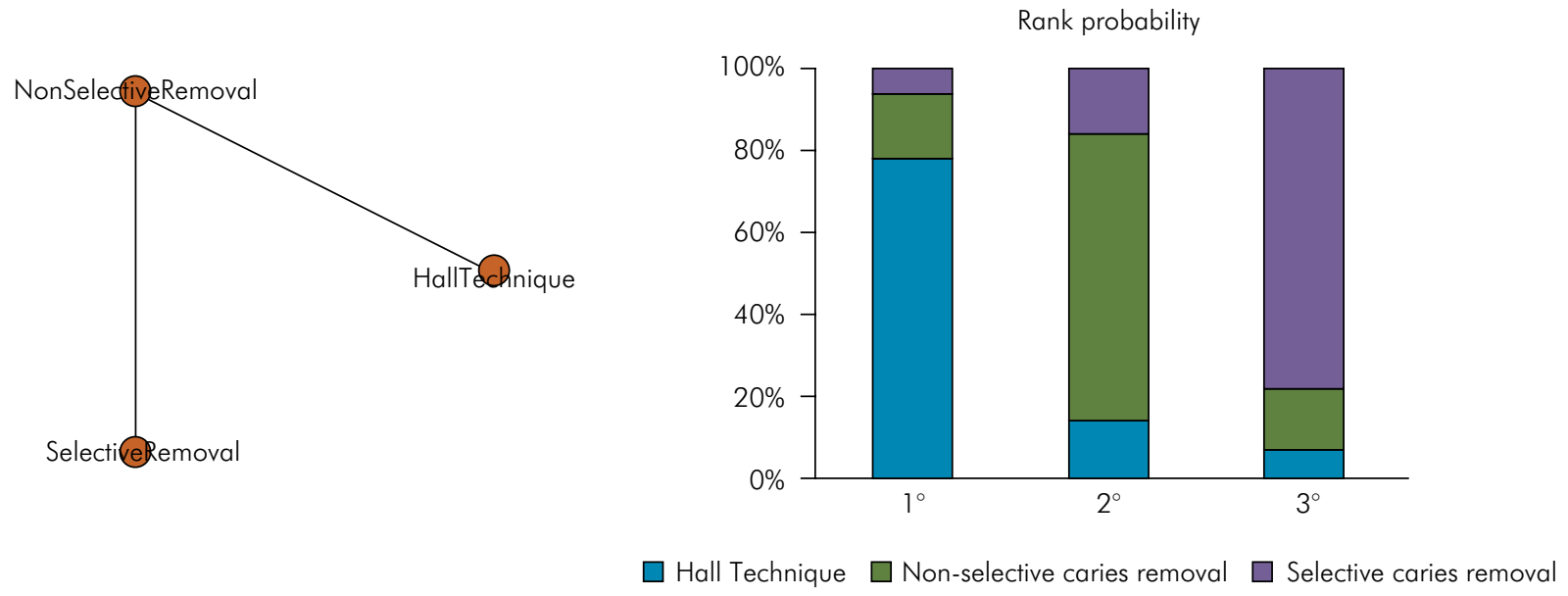

Figure 3. Network comparison and ranked success probability of treatments for deep caries lesions in vital primary teeth.

Table 4. League table with all direct* (gray) and mixed** (white) comparisons of treatments for accidental pulp exposure in the presence of vital pulp.

\begin{tabular}{lcc}
\hline Pulpectomy & $1.18(1.02-1.36)$ & \\
\hline $1.7(0.53-6.3)$ & Pulpotomy & Direct pulp capping \\
$4.5(0.36-69.0)$ & $2.6(0.27-30.0)$ & \\
\hline
\end{tabular}

Comparisons between treatments should be read from left to right. Results are OR $(95 \mathrm{Cl} \%)$, where $\mathrm{OR}>1$ favors the cell-defined treatment; *Random effect model: Mantel-Haenszel method; DerSimonian-Laird estimator for tau ${ }^{\wedge} 2 ;\left.\right|^{\wedge} 2=52 \% ; p=0.15 ;{ }^{* *}$ Random effect model, Model fit: residual deviance; DIC: 11.53.
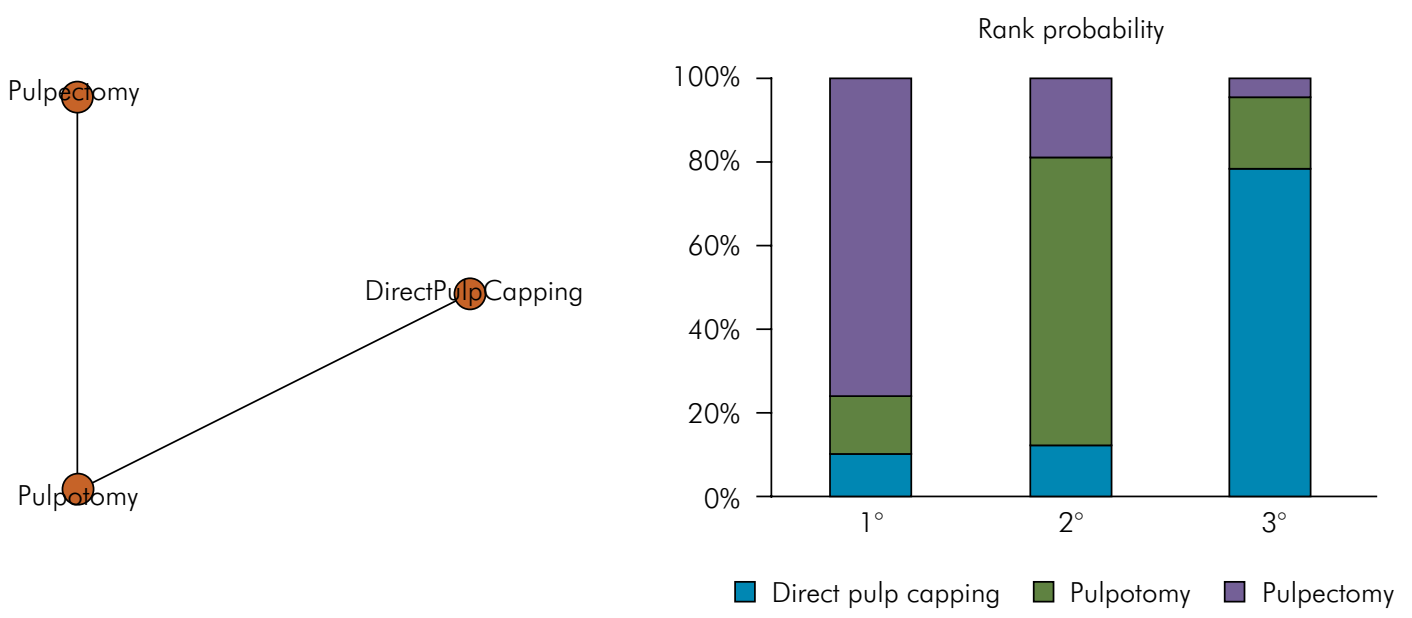

Figure 4. Network comparisons and ranked success probability of treatments for vital pulp accidentally exposed during the caries removal.

non-significant, heterogeneity was observed among included studies ( $\left.\mathrm{p}=1.007 ; \mathrm{I}^{2}=17 \%\right)$. Table 4 shows the results of direct and indirect comparisons. The rank probability showed that pulpectomy had a $76 \%$ chance of presenting the best results when used
( $1^{\text {st }}$ place), followed by pulpotomy $\left(2^{\text {nd }}\right.$ place). At the same time, direct pulp capping presented a $78 \%$ chance of having the worst performance (Figure 4).

Similarly, when irreversible pulpitis or pulp necrosis was the initial pulp condition, two studies 


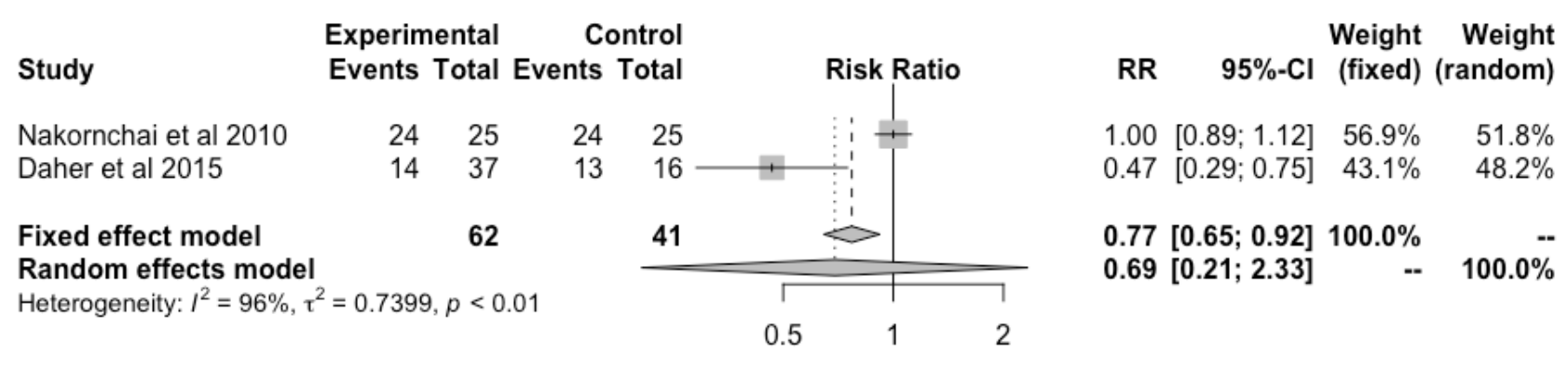

Figure 5. Meta-analyses of treatments for irreversible pulpitis and pulp necrosis comparing pulpectomy (control) with noninstrumented endodontic treatment (experimental).

were included and two different treatments were compared. Data from 12 months of follow up were considered. High and significant heterogeneity was observed among included studies ( $\left.\mathrm{p}<0.001 ; \mathrm{I}^{2}=96 \%\right)$. The meta-analysis, considering the random-effects model, was then conducted and showed no difference between pulpectomy and NIET for this pulp condition (Figure 5).

\section{Discussion}

The search for the best approach for the management of deep cavitated caries lesions has resulted in numerous treatment possibilities, which presents a challenge for pediatric dentists when having to select the best option. With this in mind, the present systematic review evaluated the clinical success of treatments for cavitated caries lesions in primary teeth with possible pulp involvement. Overall, we confirmed that the initial pulp health status is essential when choosing the adequate treatment.

When vital primary molars with deep caries were considered, the probability rank from network meta-analysis showed that the Hall Technique surpassed the other restorative techniques, such as non-selective and selective caries removal. Although few studies have considered this technique for deep caries lesions, the results found corroborate a previous systematic review focusing on cavitated caries without proximity to pulp..$^{5}$ It is therefore believed that the same phenomena previously mentioned are responsible for the higher probability of the Hall Technique resulting in clinical success. The sealing of the lesion with stainless-steel crowns hampers contact with biofilm from a dietary carbohydrate source, limiting caries progression. ${ }^{21,22}$ In addition, the use of glass ionomer cement to cement the crowns could remineralize the caries lesion, even though caries removal is not recommended in this technique ${ }^{23}$ to avoid accidental pulp exposure, maintaining the dentin-pulp complex intact and increasing pulp survival. ${ }^{12}$ However, this finding is supported by one quasi-randomized study. ${ }^{12}$ Therefore, there is a possible risk of selection bias and no similar characteristics for the experimental groups at baseline. The authors explain that the study design is because the treatment allocation was done according to the operators (dental therapists), who were responsible for selecting the participants.

Non-selective caries removal was the second best option to treat deep caries lesions without pulp involvement. However, this approach should not be considered. Despite the longevity of restoration that could be expected with non-selective caries removal, the disadvantages of this technique advise against its use. ${ }^{21,24}$ Based on the scientific evidence built to date, the recommendations are the use of less invasive techniques, such as selective caries removal, which reduces the risk of pulp exposure and its sequelae. ${ }^{21,24}$

Even in a worst-case scenario, the use of pulpotomy and pulpectomy, despite being considered in two studies, ${ }^{14,15}$ should not be a treatment option for deep caries lesions without pulp involvement. Although data from these studies was not used in the network meta-analysis due to the absence of common groups, it needs to be emphasized the discouragement of this technique in such cases for the reasons explained above..$^{21,24}$ 
Conversely, for the treatment of cavitated caries lesions with accidental pulp exposure, the network meta-analysis showed better results when a pulpectomy was performed compared with a pulpotomy and direct pulp capping. 16,17,18 Pulpotomies and direct pulp capping have been indicated in cases of accidental exposure of vital pulp or reversible pulpitis without evidence of radicular pathology, $16,18,25$ as seen in the eligibility criteria of the included studies. However, two theories could help explain the better results obtained with a pulpectomy: a) the difficulty in diagnosing pulp health condition in children leads to limitations and inaccuracies in the diagnosis and incorrect choice of technique; and b) possible technical errors such as mechanical pressure on incompletely removed coronary pulp. ${ }^{16}$ Thus, even though a pulpotomy has the objective of maintaining radicular pulp vitality, clinical success rates ranged from $63 \%$ to $86.9 \%$ compared to $95.6 \%$ to $100 \%$ for the pulpectomy. In addition, the moderate certainty of evidence in favor of pulpectomy reinforces this finding. Poor success rates can be observed also for direct pulp capping when compared with pulpotomy. ${ }^{19}$ High occurrence of internal resorption has been speculated as the reason for the unsatisfactory results of this technique. ${ }^{26}$ Possible complications such as mobility, percussion sensitivity, swelling, parulis, or the presence of a fistulous tract can result from a failed pulpotomy and direct pulp capping, ${ }^{16,17,19}$ which discourages the use of either techniques.

Furthermore, when pulpal necrosis or irreversible pulpitis were considered, no difference was observed between the treatments evaluated. Nevertheless, it is essential to highlight that this result had a very low certainty of evidence from a meta-analysis of only two studies, with high heterogeneity and wide confidence interval. ${ }^{19,20}$ While a pulpectomy associates the mechanical cleaning and chemical disinfection of the entire root canal, NIET or lesion sterilization and tissue repair therapy are intended to disinfect affected pulp and periapical tissues with an antibacterial paste, without mechanical instrumentation. ${ }^{19,20}$ Because the composition of antibacterial pastes varied greatly between the studies and of the small number of included studies, the impact of this factor could not be evaluated in the present systematic review. However, it has been suggested that treatment success appears to be more related to the initial pathological condition than the filling material for pulp treatment. ${ }^{27}$ Even so, the standardization of these variables should be considered in future studies.

In our study, we considered the clinical treatment success as an outcome. Most of the studies, with the exception of one, included clinical and radiographic success rates separately. Because there is no standard index or criteria to evaluate pulp treatments, some characteristics have been considered to evaluate treatment success: satisfactory restoration and absence of clinical symptoms and signs of pulp pathology, such as fistula, swelling, spontaneous pain, and mobility not compatible with root resorption. These clinical characteristics can be easily identified by dentists and be considered a more relevant outcome.

Finally, it is essential to emphasize that all included studies presented a high risk of bias since all had at least one domain with high risk, 12,13,14,15,16,17,18,19,20 especially selection and performance bias. However, the blinding of the operator is not always possible due to the notable difference between the techniques. Contrarily, the domains of attrition, reporting, and other biases were scored as having a low risk of bias.

In addition, the findings that the Hall Technique and pulpectomy are the best treatments for deep cavitated caries lesions with vital pulp and accidental pulp exposure, respectively, are from the ranking probability analysis. Although this network metaanalysis tool can help organize the preferential order of treatments, this information does not necessarily imply clinically relevant effect size. Thus, the results should be considered with caution. This is reinforced by the small number of studies included in the analysis, which is a limitation of our systematic review. Therefore, further well-designed studies focusing on conducting and reporting allocation concealment and blinding of participants and examiners, when possible, need to be performed to obtain a robust evidence to guide pediatric dentists in the decision-making process. 


\section{Conclusion}

The available evidence indicates the Hall Technique as having the best-ranked probability for treatment success of deep caries lesions in vital primary teeth. In cases of accidental vital pulp exposure during caries removal, pulpectomy can be considered the best option. However, the certainty of evidence ranged from very low to moderate and the analysis included a small number of studies. Furthermore, no evidence for the best treatment for irreversible pulpitis or pulp necrosis could be obtained.

\section{Acknowledgments}

The authors would like to thank Luiz Panariello Filho for assistance with statistical analysis.

\section{References}

1. Gimenez T, Bispo BA, Souza DP, Viganó ME, Wanderley MT, Mendes FM, et al. Does the decline in caries prevalence of Latin American and Caribbean children continue in the new century? Evidence from systematic review with meta-analysis. PLoS One. 2016 Oct;11(10):e0164903. https://doi.org/10.1371/journal.pone.0164903

2. Guedes RS, Ardenghi TM, Piovesan C, Emmanuelli B, Mendes FM. Influence of initial caries lesions on quality of life in preschool children: a 2-year cohort study. Community Dent Oral Epidemiol. 2016 Jun;44(3):292-300. https://doi.org/10.1111/cdoe.12217

3. Yengopal V, Harnekar SY, Patel N, Siegfried N. WITHDRAWN: dental fillings for the treatment of caries in the primary dentition. Cochrane Database Syst Rev. 2016 Oct;10:CD004483.

4. Tedesco TK, Calvo AF, Lenzi TL, Hesse D, Guglielmi CA, Camargo LB, et al. ART is an alternative for restoring occlusoproximal cavities in primary teeth - evidence from an updated systematic review and meta-analysis. Int J Paediatr Dent. 2017 May;27(3):201-9. https://doi.org/10.1111/ipd.12252

5. Tedesco TK, Gimenez T, Floriano I, Montagner AF, Camargo LB, Calvo AF, et al. Scientific evidence for the management of dentin caries lesions in pediatric dentistry: A systematic review and network meta-analysis. PLoS One. 2018 Nov;13(11):e0206296. https://doi.org/10.1371/journal.pone.0206296

6. Ladewig NM, Tedesco TK, Gimenez T, Braga MM, Raggio DP. Patient-reported outcomes associated with different restorative techniques in pediatric dentistry: A systematic review and MTC meta-analysis. PLoS One. 2018 Dec;13(12):e0208437. https://doi.org/10.1371/journal.pone.0208437

7. Schwendicke F, Brouwer F, Schwendicke A, Paris S. Different materials for direct pulp capping: systematic review and meta-analysis and trial sequential analysis. Clin Oral Investig. 2016 Jul;20(6):1121-32. https://doi.org/10.1007/s00784-016-1802-7

8. Coll JA, Seale NS, Vargas K, Marghalani AA, AI Shamali S, Graham L. Primary tooth vital pulp therapy: a systematic review and meta-analysis. Pediatr Dent. 2017 Jan;39(1):16-123.

9. Dhar V, Marghalani AA, Crystal YO, Kumar A, Ritwik P, Tulunoglu O, et al. Use of Vital Pulp Therapies in Primary Teeth with Deep Caries Lesions. Pediatr Dent. 2017 Sep;39(5):146-59.

10. Hutton B, Salanti G, Caldwell DM, Chaimani A, Schmid CH, Cameron C, et al. The PRISMA extension statement for reporting of systematic reviews incorporating network meta-analyses of health care interventions: checklist and explanations. Ann Intern Med. 2015 Jun;162(11):777-84. https://doi.org/10.7326/M14-2385

11. Higgins JP, Green S. Cochrane handbook for systematic reviews of interventions version 5.0.1 [updated September 2008]. London: The Cochrane Collaboration; 2008 [cited year Month day]. Available from www.cochrane-handbook.org

12. Boyd DH, Page LF, Thomson WM. The Hall technique and conventional restorative treatment in New Zealand children's primary oral health care: clinical outcomes at two years. Int J Paediatr Dent. 2018 Mar;28(2):180-8. https://doi.org/10.1111/ipd.12324

13. Franzon R, Guimarães LF, Magalhães CE, Haas AN, Araujo FB. Outcomes of one-step incomplete and complete excavation in primary teeth: a 24 -month randomized controlled trial. Caries Res. 2014;48(5):376-83. https://doi.org/10.1159/000357628

14. Howley B, Seale NS, McWhorter AG, Kerins C, Boozer KB, Lindsey D. Pulpotomy versus pulpectomy for carious vital primary incisors: randomized controlled trial. Pediatr Dent. 2012 Sep-Oct;34(5):112-9.

15. Tang $Y, X u$ W. Therapeutic effects of Pulpotomy and Pulpectomy on deciduous molars with deep caries. Pak J Med Sci. 2017 Nov-Dec;33(6):1468-72. https://doi.org/10.12669/pims.336.13488 PMID:29492080

16. Aminabadi NA, Farahani RM, Gajan EB. A clinical study of formocresol pulpotomy versus root canal therapy of vital primary incisors. J Clin Pediatr Dent. 2008;32(3):211-4. https://doi.org/10.17796/jcpd.32.3.ghk26v4554790074

17. Casas MJ, Kenny DJ, Johnston DH, Judd PL, Layug MA. Outcomes of vital primary incisor ferric sulfate pulpotomy and root canal therapy. J Can Dent Assoc. 2004 Jan;70(1):34-8. 
Management of deep caries lesions with or without pulp involvement in primary teeth: a systematic review and network meta-analysis

18. Dimitraki D, Papageorgiou SN, Kotsanos N. Direct pulp capping versus pulpotomy with MTA for carious primary molars: a randomised clinical trial. Eur Arch Paediatr Dent. 2019 Oct;20(5):431-40. https://doi.org/10.1007/s40368-019-00419-7

19. Daher A, Viana KA, Leles CR, Costa L. Ineffectiveness of an antibiotic-based pulpotomy for primary molars: a survival analysis. Pesqui Bras Odontopediatria Clin Integr. 2015;15(1):205-15. https://doi.org/10.4034/PBOCl.2015.151.22.

20. Nakornchai S, Banditsing P, Visetratana N. Clinical evaluation of $3 \mathrm{Mix}$ and Vitapex as treatment options for pulpally involved primary molars. Int J Paediatr Dent. 2010 May;20(3):214-21. https://doi.org/10.1111/j.1365-263X.2010.01044.x

21. Innes NP, Chu CH, Fontana M, Lo EC, Thomson WM, Uribe S, et al. A century of change towards prevention and minimal intervention in cariology. J Dent Res. 2019 Jun;98(6):611-7. https://doi.org/10.1177/0022034519837252

22. Mijan M, Amorim RG, Leal SC, Mulder J, Oliveira L, Creugers NH, et al. The 3.5-year survival rates of primary molars treated according to three treatment protocols: a controlled clinical trial. Clin Oral Investig. 2014 May; 18(4):1061-9. https://doi.org/10.1007/s00784-013-1077-1 .

23. Innes NP, Evans DJ, Stirrups DR. Sealing caries in primary molars: randomized control trial, 5-year results. J Dent Res. 2011 Dec;90(12):1405-10. https://doi.org/10.1177/0022034511422064

24. Schwendicke F, Frencken J, Innes N. Clinical Recommendations on Carious Tissue Removal in Cavitated Lesions. Monogr Oral Sci. 2018;27:162-6. https://doi.org/10.1159/000487843

25. American Academy of Pediatric Dentistry - AAPD. Pulp therapy for primary and immature permanent teeth. Pediatr Dent. 2017 Sep;39(6):325-33.

26. Sujlana A, Pannu PK. Direct pulp capping: a treatment option in primary teeth? Pediatr Dent J. 2017;27(1):1-7. https://doi.org/10.1016/i.pdi.2016.10.001

27. Barcelos R, Santos MP, Primo LG, Luiz RR, Maia LC. ZOE paste pulpectomies outcome in primary teeth: a systematic review. J Clin Pediatr Dent. 2011;35(3):241-8. https://doi.org/10.17796/jcpd.35.3.y777187463255n34 